\title{
Exact Results for the Homogenization of Elastic Fiber-Reinforced Solids at Finite Strain
}

\author{
Q.-C. HE ${ }^{1}$, H. LE QUANG ${ }^{1}$ and Z.-Q. FENG ${ }^{2}$ \\ ${ }^{1}$ Laboratoire de Mécanique, Université de Marne-la-Vallée, 19 rue A. Nobel, \\ 77420 Champs sur Marne, France.E-mail: he@univ-mlv.fr \\ ${ }^{2}$ Laboratoire de Mécanique d'Evry, Université d'Evry - Val d'Essonne, 40 rue du Pelvoux, \\ 91020 Evry, France.
}

\begin{abstract}
This work is concerned with the homogenization of solids reinforced by aligned parallel continuous fibers or weakened by aligned parallel cylindrical pores and undergoing large deformations. By alternatively exploiting the nominal and material formulations of the corresponding homogenization problem and by applying the implicit function theorem, it is shown that locally homogeneous deformations can be produced in such inhomogeneous materials and form a differentiable manifold. For every macroscopic strain associated to a locally homogeneous deformation field, the effective nominal or material stress-strain relation is exactly determined and connections are also exactly established between the effective nominal and material elastic tangent moduli. These results are microstructure-independent in the sense that they hold irrespectively of the transverse geometry and distribution of the fibers or pores. A porous medium consisting of a compressible Mooney-Rivlin material with cylindrical pores is studied in detail to illustrate the general results.
\end{abstract}

Mathematics Subject Classifications (2000): 74B20, 74Q15.

Key words: homogenization, fiber-reinforced composites, porous materials, homogeneous strain fields, microstructure, large deformations.

\section{Introduction}

For the prediction of the effective mechanical properties of linear inhomogeneous or composite materials, micromechanical theories have been substantially developed and numerous general and specific results are now available (see [22, $26,32]$ ). A considerable effort has also been devoted in recent years to estimate or bound the effective behavior of nonlinear composite materials subjected to infinitesimal strains (see, e.g., [23, 30, 31]). By contrast, the homogenization of composite materials undergoing large deformations has been little investigated,

This work was the first time presented at the Euromech Colloqium 464 on "Fiber-reinforced Solids: Constitutive Laws and Instabilities," September 28-October 1, 2004, Cantabria, Spain. 
although a framework was provided by Hill [15] more than 30 years ago and although interesting results were obtained in some general or particular situations (see, e.g., [10, 18, 19, 25, 27, 28]). The present work is concerned with the homogenization of elastic fiber-reinforced composite materials at finite strain, aiming to furnish a few exact results for this problem. In particular, by considering aligned parallel cylindrical pores in place of aligned parallel fibers, the present work is also relative to the homogenization of a class of porous materials.

Within the framework of linear elasticity, Hill [14] discovered that the effective elastic moduli of a transversely isotropic fiber-reinforced composite with two transversely isotropic phases are connected by two exact relations independent of the transverse geometry and arrangement of the fibers at given volume fraction, so that the usual number of five independent elastic moduli for a transversely isotropic material reduces to three. This important result was extended by Dvorak [7] to totally anisotropic linear fiber-reinforced composites through systematically studying and exploiting the concept of homogeneous deformations in inhomogeneous materials. Inspired by the works of Hill [14] and Dvorak [7], He [12] generalized their conclusions to nonlinear elastic fiberreinforced composites subjected to infinitesimal strains by using the implicit function theorem of mathematical analysis. The present work poses and answers the following question: Is it possible to further extend the results of Hill [14] and Dvorak [7] to elastic fiber-reinforced composites undergoing large deformations?

As will be seen, the key to giving a definite answer to the previous question lies in applying the implicit function theorem. However, a dilemma is encountered in the context of finite elasticity. On one side, as pointed by Hill [15] (see also [25]), for the transition from microvariables to macrovariables, the deformation gradient tensor and the nominal (or first Piola-Kirchhoff) stress tensor are the physically meaningful couple of kinematical and static variables. On the other hand, for the principle of objectivity [34] to be satisfied, the material (or Green-Lagrange) strain tensor and the material (or second PiolaKirchhoff) stress tensor constitute a much more convenient couple of kinematical and static variables. This conflict is circumvented by alternate use of the nominal and material descriptions in solving the problem addressed in the present work.

In the context of finite elasticity, the treatment of homogenization problems must account for the instability phenomena due to the fact that the strain-energy function cannot be convex with respect to the deformation gradient tensor (see, e.g., $[2,5])$. Within the framework of periodic hyperelastic composites, a few authors, including Abeyaratne and Triantafyllidis [1], Triantafyllidis and Maker [33], Müller [24], Geymonat et al. [8] and Miehe et al. [21], have at different levels of generality studied the microscopic and macroscopic instabilities as well as their relations. In particular, considering a Neo-Hookean matrix with periodic cylindrical pores, Abeyaratne and Triantafyllidis [1] found that the homogenized 
tangential tensor of the porous material loses strong ellipticity while the matrix material remains strongly elliptic. Examining a specific class of fiber-reinforced layered periodic composites, Triantafyllidis and Maker [33] shown that the bifurcation of such a composite at a wavelength much larger than the unit cell size is associated with the loss of strong ellipticity of the homogenized tangential tensor. The relations between microscopic and macroscopic instabilities were further investigated by Geymonat et al. [8] in the context of non-convex functional analysis, who, in a rather general way, demonstrated that long wavelength instabilities for the linearized problem lead to the loss of rank-one convexity of the homogenized strain-energy function. As pointed out by Müller [24], a major difficulty due to the occurrence of microscopic instabilities is that the representative volume element (RVE) used to formulate the homogenization problem does not a priori correspond to the unit cell of a periodic composite. The RVE must contain a sufficient number of unit cells so as to catch the energyminimizing modes (see $[20,21]$ for more details). In the present wok, we do not deal with any instability issue relative to the class of fiber-reinforced composites under consideration, although instability phenomena may occur at the microscopic and macroscopic levels. In fact, our work does not require that fibers be arranged periodically in the transverse plane, so that a random transverse distribution of fibers is not excluded. Except the recent one of Lopez-Pamies and Ponte Castañeda [19], the works reported in the literature on microscopic and macroscopic phenomena related to the homogenization of composites at finite strain are concerned almost exclusively with periodic microstructures. The relevant instability issue in the case of random microstructures remains largely open and is far beyond the scope of the present work. The results presented in this paper on the existence of homogeneous deformation fields and the exact relations should be taken to be valid only until microscopic instabilities appear.

The paper is organized as follows. Section 2 is dedicated to the specification and formulation of the problem to be dealt with. In Section 3, while requiring the principle of objectivity to be checked, the implicit function theorem is applied to show that material and nominal homogeneous deformation fields can be generated in fiber-reinforced composites and form two differential manifolds which are related by the rotation group. In Section 4, the effective elastic nominal and material stress-strain relations are exactly determined for all macroscopic deformations associated to a locally homogeneous deformation field. General exact connections are also established between the effective elastic nominal and material tangent moduli evaluated at each macroscopic strain inducing a locally homogeneous deformation field. The results of Section 4 are illustrated in Section 5 through an example where a compressible MooneyRivlin material weakened by aligned parallel cylindrical pores is considered. The results obtained in the present paper are independent of the transverse morphology and distribution of the fibers at given volume fraction, as in the 
case of linear elasticity. These results serve directly for partial determination of the effective elastic properties of fiber-reinforced composites undergoing large deformations and can be also used as benchmarks for analytical and numerical approximate methods elaborated to estimate them.

Coordinate-free notation is adopted in the present paper. As a general rule, light-face (Greek or Latin) letters designate scalars, sets, spaces, domains or groups. Bold-face lowercase and uppercase Latin letters represent vectors and second-order tensors, respectively. Outline Latin letters are reserved for fourthorder tensors. The components of a vector, second- or fourth-order tensor are represented by the corresponding light-face letter with a suitable number of subscripts. Further, we denote by $R^{3}$ the usual three-dimensional (3D) Euclidian space, by Lin the space of all second-order tensors on $R^{3}$, by Sym the subspace of all second-order symmetric tensors on $R^{3}$, and by $\operatorname{Lin}^{+}$the cone of all second-order positive-determinant tensors belonging to Lin. Given any two elements $\mathbf{A}$ and $\mathbf{B}$ of Lin, we define, apart from the usual tensor product $\mathbf{A} \otimes$ $\mathbf{B}$, two tensor products $\mathbf{A} \otimes \mathbf{B}$ and $\mathbf{A} \bar{\otimes} \mathbf{B}$ of Kronecker type (see, e.g., [13]) by

$$
(\mathbf{A} \underline{\otimes} \mathbf{B})(\mathbf{u} \otimes \mathbf{v})=(\mathbf{A u}) \otimes(\mathbf{B v}), \quad(\mathbf{A} \bar{\otimes} \mathbf{B})(\mathbf{u} \otimes \mathbf{v})=(\mathbf{A v}) \otimes(\mathbf{B u})
$$

for any two elements $\mathbf{u}$ and $\mathbf{v}$ of $R^{3}$. It is also convenient to introduce another tensor product $\mathbf{A} \underline{\bar{\otimes}} \mathbf{B}$ by

$$
\mathbf{A} \underline{\bar{\otimes}} \mathbf{B}=\frac{1}{2}(\mathbf{A} \underline{\otimes} \mathbf{B}+\mathbf{A} \bar{\otimes} \mathbf{B}) .
$$

Next, the fourth-order identity tensor $\mathbb{I}$ on Lin, the transposition mapping $\mathbb{T}$ on Lin, and the fourth-order identity tensor $\mathbb{1}$ on Sym can be expressed in terms of the second-order identity tensor $\mathbf{I}$ as follows:

$$
\mathbb{I}=\mathbf{I} \underline{\otimes} \mathbf{I}, \quad \mathbb{T}=\mathbf{I} \bar{\otimes} \mathbf{I}, \quad \mathbb{1}=\underline{\mathbf{I}} \underline{\bar{\otimes}} \mathbf{I} .
$$

For later use, remark that

$$
(\mathbf{A} \underline{\otimes} \mathbf{B}) \mathbf{X}=\mathbf{A} \mathbf{X} \mathbf{B}^{T}, \quad(\mathbf{A} \bar{\otimes} \mathbf{B}) \mathbf{X}=\mathbf{A} \mathbf{X}^{T} \mathbf{B}^{T}, \quad(\mathbf{A} \underline{\otimes} \mathbf{B}) \mathbf{X}=\frac{1}{2} \mathbf{A}\left(\mathbf{X}+\mathbf{X}^{T}\right) \mathbf{B}^{T}
$$

for any three elements $\mathbf{A}, \mathbf{B}$ and $\mathbf{X}$ of Lin. In fact, the matrix components forms of $\mathbf{A} \underline{\otimes} \mathbf{B}, \mathbf{A} \bar{\otimes} \mathbf{B}$ and $\mathbf{A} \bar{\otimes} \mathbf{B}$ are given by $(\mathbf{A} \underline{\otimes} \mathbf{B})_{i j k l}=\mathrm{A}_{i k} \mathrm{~B}_{j l}, \quad(\mathbf{A} \bar{\otimes} \mathbf{B})_{i j k l}=$ $\mathbf{A}_{i l} \mathbf{B}_{j k}$ and $(\mathbf{A} \underline{\otimes} \mathbf{B})_{i j k l}=\frac{1}{2}\left(\overline{\mathrm{A}}_{i k} \mathrm{~B}_{j l}+\mathrm{A}_{i l} \mathrm{~B}_{j k}\right)$.

\section{Setting of the Problem}

In this section, we describe and formulate the problem with which the present work is concerned. 


\subsection{LOCAL STRESS-STRAIN RELATIONS AND MACROSCOPIC VARIABLES}

The inhomogeneous material investigated in this work consists of a solid matrix reinforced by aligned parallel continuous fibers (see Figure 1). The matrix and fiber phases are assumed to be individually homogeneous and perfectly bonded together across interfaces but no restrictions are imposed on the transverse geometry and distribution of the fibers, which may be random. The two-phase composite under consideration is thus homogeneous along the fiber direction while being inhomogeneous in the transverse plane. Let $\Omega$ be the domain occupied by a specimen of the composite in the reference configuration and let $\Omega^{(r)}$ correspond to the sub-domain of phase $r(=1,2)$. In what follows, we designate the boundary of $\Omega$ by $\partial \Omega$, the phase interfaces by $\Gamma$, the volume average over $\Omega$ by $<\cdot>$, the volume average over $\Omega^{(r)}$ by $<\cdot>_{r}$, and the initial volume fraction of phase $r$ by $c^{(r)}$. For definiteness, the matrix is referred to as phase 1 , the fibers are called phase 2 , and the direction of the fibers is defined by the unit vector $\mathbf{n}$ (see Figure 1).

The local (or microscopic) behavior of each phase of the composite is assumed to be nonlinearly elastic. Denoting by $\mathbf{F}$ the gradient of a deformation $\mathbf{y}: \mathbf{x} \in \Omega \mapsto \mathbf{y}(\mathbf{X}) \in R^{3}$, and by $\mathbf{P}$ the resulting nominal stress, the local elastic stress-strain relation of the composite is described by

$$
\mathbf{P}=\mathbf{P}(\mathbf{x}, \mathbf{F})=\sum_{r=1}^{r=2} \chi^{(r)}(\mathbf{x}) \mathbf{P}^{(r)}(\mathbf{F}) .
$$

Here, $\mathbf{P}^{(r)}(\mathbf{F})$ is the nonlinear stress-strain relation of phase $r$ and $\chi^{(r)}$ is the characteristic function of $\Omega^{(r)}$ such that $\chi^{(r)}(\mathbf{x})=1$ for $\mathbf{x} \in \Omega^{(r)}$ and $\chi^{(r)}(\mathbf{x})=0$ for $\mathbf{x} \notin$ $\Omega^{(r)}$. The characteristic functions $\chi^{(r)}$ entirely describe the microstructure of the composite. The elastic stress-strain relation (2.1) is required to satisfy the prin-

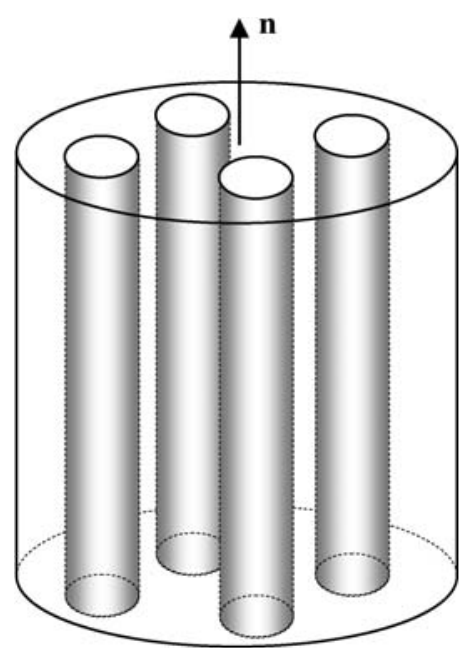

Figure 1. A fiber-reinforced composite or unidirectional pore-weakened material. 
ciple of objectivity and the relevant material symmetry conditions [34]. Designating the $3 \mathrm{D}$ rotation group by $\mathrm{SO}(3)$, the principle of objectivity reads

$$
\mathbf{P}^{(r)}(\mathbf{Q F})=\mathbf{Q} \mathbf{P}^{(r)}(\mathbf{F}), \quad \forall \mathbf{Q} \in S O(3), \quad \forall \mathbf{F} \in \operatorname{Lin}^{+} .
$$

If the material symmetry of phase $r$ is specified by $G^{(r)} \subseteq S O(3)$, then $\mathbf{P}^{(r)}(\mathbf{F})$ must satisfy the condition

$$
\mathbf{P}^{(r)}(\mathbf{F Q})=\mathbf{P}^{(r)}(\mathbf{F}) \mathbf{Q}, \quad \forall \mathbf{Q} \in G^{(r)}, \quad \forall \mathbf{F} \in \operatorname{Lin}^{+} .
$$

In this paper, the particularization of $G^{(r)}$ is not necessary. However, use will be explicitly or tacitly made of the following necessary and sufficient condition for (2.2) to be verified:

$$
\mathbf{P}^{(r)}(\mathbf{F})=\mathbf{R} \mathbf{P}^{(r)}(\mathbf{U}), \quad \forall \mathbf{F} \in \operatorname{Lin}^{+},
$$

where $\mathbf{R}$ and $\mathbf{U}$ are the respective rotation and right stretch tensors in the polar decomposition $\mathbf{F}=\mathbf{R U}$.

In what follows, we make the additional assumption that the stress-strain relation $\mathbf{P}^{(r)}(\mathbf{F})$ of every phase is continuously differentiable and verifies the initial condition

$$
\mathbf{P}^{(r)}(\mathbf{I})=\mathbf{0} .
$$

As will be seen, the continuous differentiability of $\mathbf{P}^{(r)}(\mathbf{F})(r=1,2)$ is essential to the present work while the condition (2.5), which means that phase $r$ is free of residual stress in the reference configuration, can be relaxed.

Alternatively, the local behavior of the composite can be described by

$$
\mathbf{S}=\mathbf{S}(\mathbf{x}, \mathbf{E})=\sum_{r=1}^{r=2} \chi^{(r)}(\mathbf{x}) \mathbf{S}^{(r)}(\mathbf{E}) .
$$

Here, $\mathbf{E}$ is the Green-Lagrange strain tensor defined by

$$
\mathbf{E}=\frac{1}{2}(\mathbf{C}-\mathbf{I}) \quad \text { with } \quad \mathbf{C}=\mathbf{F}^{T} \mathbf{F}=\mathbf{U}^{2},
$$

and $\mathbf{S}$ is the material stress tensor. The material stress-strain relation $\mathbf{S}^{(r)}(\mathbf{E})$ of phase $r$ is related to its nominal one $\mathbf{P}^{(r)}(\mathbf{F})$ by

$$
\mathbf{P}^{(r)}(\mathbf{F})=\mathbf{F S}^{(r)}(\mathbf{E}) .
$$

With (2.1), the material symmetry requirement (2.3) and the residual-stress free condition (2.5) become

$$
\begin{aligned}
& \mathbf{S}^{(r)}\left(\mathbf{Q E} \mathbf{Q}^{T}\right)=\mathbf{Q S}^{(r)}(\mathbf{E}) \mathbf{Q}^{T}, \quad \forall \mathbf{Q} \in G^{(r)}, \quad \forall \mathbf{E} \in \text { Sym }, \\
& \mathbf{S}^{(r)}(\mathbf{0})=\mathbf{0} .
\end{aligned}
$$


It is known from continuum mechanics (see, e.g., [9]) that the main advantage of using the material stress-strain relation (2.6) in place of the nominal one (2.1) is that the principle of objectivity is automatically satisfied by the former. However, from the standpoint of micromechanics, the conjugated pair $(\mathbf{P}, \mathbf{F})$ is more appropriate. Indeed, the macroscopic nominal stress $\overline{\mathbf{P}}$ and deformation gradient $\overline{\mathbf{F}}$ are simply the volume averages of their microscopic counterparts over $\Omega$, which are dependent uniquely on the surface tractions and displacements on $\partial \Omega[15]$ :

$$
\begin{aligned}
& \overline{\mathbf{F}}=<\mathbf{F}>=\frac{1}{|\Omega|} \int_{\Omega} \mathbf{F d v}=\frac{1}{|\Omega|} \int_{\partial \Omega} \mathbf{y} \otimes \mathbf{x d v} \\
& \overline{\mathbf{P}}=<\mathbf{P}>=\frac{1}{|\Omega|} \int_{\Omega} \mathbf{P d v}=\frac{1}{|\Omega|} \int_{\partial \Omega}(\mathbf{P m}) \otimes \mathbf{x d v},
\end{aligned}
$$

where $\mathbf{m}$ is the outward unit vector normal to $\partial \Omega$. Then, the macroscopic GreenLagrange strain tensor $\overline{\mathbf{E}}$ and material stress tensor $\overline{\mathbf{S}}$ are obtained through $\overline{\mathbf{F}}$ :

$$
\begin{aligned}
& \overline{\mathbf{E}}=\frac{1}{2}\left(\overline{\mathbf{F}}^{T} \overline{\mathbf{F}}-\mathbf{I}\right)=\frac{1}{2}\left(<\mathbf{F}>^{T}<\mathbf{F}>-\mathbf{I}\right), \\
& \overline{\mathbf{S}}=\overline{\mathbf{F}}^{-1} \overline{\mathbf{P}}=\left\langle\mathbf{F}>^{-1}<\mathbf{P}>.\right.
\end{aligned}
$$

It is important to remark that, in general, $\overline{\mathbf{E}} \neq<\mathbf{E}>$ and $\overline{\mathbf{S}} \neq<\mathbf{S}>$ (see Hill [15]).

\subsection{FORMULATION OF THE HOMOGENIZATION PROBLEM}

Even though no limitations are imposed on the precise geometrical forms of the transverse cross-sections of the fibers and on the precise way in which the fibers are arranged in the transverse plane, we require that their transverse distribution be statistically uniform and that the largest dimension of the transverse crosssection of each fiber be much smaller than the dimensions of the specimen $\Omega$. These conditions make it meaningful to consider $\Omega$ as an RVE and investigate the homogenization problem related to the composite.

Since the phases are nonlinearly elastic and perfectly bonded together, the effective (or macroscopic) behavior of the composite is also nonlinearly elastic and can be described by the effective nominal stress-strain relation

$$
\overline{\mathbf{P}}=\hat{\mathbf{P}}(\overline{\mathbf{F}})
$$

The homogenization problem under consideration is to determine the effective stress-strain function $\hat{\mathbf{P}}(\overline{\mathbf{F}})$ from the knowledge of the microstructure character- 
ized by $\chi^{(r)}$ and the phase stress-strain relations specified by $\mathbf{P}^{(r)}(\mathbf{F})$. Together with the definition (2.12) of $\overline{\mathbf{P}}$, this is a boundary value problem of nonlinear inhomogeneous elasticity, which can be formulated as follows.

Without loss of generality, consider the case where a linear transformation is prescribed on the boundary $\partial \Omega$ of $\Omega$ :

$$
\mathbf{y}(\mathbf{x})=\overline{\mathbf{F}} \mathbf{x} \text { on } \partial \Omega
$$

where $\overline{\mathbf{F}}$ is a given second-order tensor belonging to $\operatorname{Lin}^{+}$. Due to the surface loading (2.16), a deformation field $\mathbf{y}$, a deformation gradient field $\mathbf{F}$ and a nominal stress field $\mathbf{P}$ are produced over $\Omega$. From now on, it is convenient to denote the restriction of $\mathbf{y}, \mathbf{F}$ and $\mathbf{P}$ to phase $r$ as $\mathbf{y}^{(r)}, \mathbf{F}^{(r)}$ and $\mathbf{P}^{(r)}$. In addition to the boundary condition (2.16) and to the local stress-strain law (2.1), $\mathbf{y}, \mathbf{F}$ and $\mathbf{P}$ must verify the following field equations and perfect interface conditions:

- the equation relating $\mathbf{F}^{(r)}$ to $\mathbf{y}^{(r)}$,

$$
\mathbf{F}^{(r)}=\nabla \mathbf{y}^{(r)} \quad \text { over } \quad \Omega^{(r)}
$$

- the equilibrium of forces,

$$
\operatorname{Div} \mathbf{P}^{(r)}=\mathbf{0} \quad \text { over } \Omega^{(r)} ;
$$

- the equilibrium of moments,

$$
\mathbf{P}^{(r)} \mathbf{F}^{(r) T}=\mathbf{F}^{(r)} \mathbf{P}^{(r) T} \quad \text { over } \quad \Omega^{(r)}
$$

- the deformation continuity across the interface,

$$
\mathbf{y}^{(2)}-\mathbf{y}^{(1)}=\mathbf{0} \quad \text { on } \quad \Gamma
$$

- the stress continuity conditions across the interface,

$$
\left(\mathbf{P}^{(2)}-\mathbf{P}^{(1)}\right) \mathbf{N}^{\perp}=\mathbf{0} \text { on } \Gamma .
$$

In (2.18), body forces have been neglected. In (2.21), $\mathbf{n}^{\perp}$ is any unit vector normal to the interface $\Gamma$ between the matrix and the fibers in the reference configuration $\Omega$ of the composite. Since the direction of the fibers is defined by the unit vector $\mathbf{n}$, it follows that $\mathbf{n}^{\perp}$ is characterized by $\mathbf{n}^{\perp} \cdot \mathbf{n}=0$.

The complete determination of the effective stress-strain relation (2.15) requires solving the nonlinear boundary value problem formulated by (2.16)(2.21) together with (2.1). This problem is a very difficult one, since it is governed by a system of highly nonlinear partial differential equations with random coefficients. Consequently, it can be generally treated only numerically. However, as it will be shown below, using the fact that the composite is homogeneous along the fiber direction $\mathbf{n}$, there are some special forms for the 
deformation gradient tensor $\overline{\mathbf{F}}$ in (2.16), such that the aforementioned nonlinear boundary value problem admits some particular homogeneous strain fields as exact solutions.

To close this section, we remark that the homogenization of materials with unidirectional cylindrical pores can be considered as a special case of the problem formulated above. Indeed, it suffices to set $\mathbf{P}^{(2)}=\mathbf{0}$ or $\mathbf{S}^{(2)}=\mathbf{0}$.

\section{Homogeneous Elastic Deformation Fields}

Consider a homogeneous deformation field

$$
\mathbf{y}(\mathbf{x})=\mathbf{F}^{*} \mathbf{x} \quad \text { over } \Omega
$$

where $\mathbf{F}^{*} \in \operatorname{Lin}^{+}$. Then, (2.18), (2.20) and (2.16) with $\overline{\mathbf{F}}=\mathbf{F}^{*}$ are immediately verified. Accounting for (2.1) and (2.17), satisfaction of the remaining equations, i.e., (2.19) and (2.21), requires that

$$
\begin{gathered}
{\left[\mathbf{P}^{(2)}\left(\mathbf{F}^{*}\right)-\mathbf{P}^{(1)}\left(\mathbf{F}^{*}\right)\right] \mathbf{n}^{\perp}=\mathbf{0} \quad \text { on } \quad \Gamma,} \\
\mathbf{P}^{(r)}\left(\mathbf{F}^{*}\right) \mathbf{F}^{* T}=\mathbf{F}^{*} \mathbf{P}^{(r) T}\left(\mathbf{F}^{*}\right) \quad \text { over } \quad \Omega^{(r)} .
\end{gathered}
$$

These two equations constitute the nominal necessary and sufficient conditions for the existence of homogeneous elastic deformations in the composite. Note that (3.2) must hold for any unit vector $\mathbf{n}^{\perp}$ perpendicular to the fiber direction $\mathbf{n}$. Thus, (3.2) can be conveniently written in the following equivalent form:

$$
[\underline{\mathbf{I} \otimes}(\mathbf{I}-\mathbf{N})]\left[\mathbf{P}^{(2)}\left(\mathbf{F}^{*}\right)-\mathbf{P}^{(1)}\left(\mathbf{F}^{*}\right)\right]=\mathbf{0},
$$

where $\mathbf{N}=\mathbf{n} \otimes \mathbf{n}$. Introducing the jump operator $\llbracket \bullet \rrbracket$ across the interface $\Gamma$ such that $\llbracket \bullet \rrbracket=\bullet^{(2)}-\bullet^{(1)}$, we can further write (3.4) as $[\mathbf{I} \underline{\otimes}(\mathbf{I}-\mathbf{N})] \llbracket \mathbf{P}\left(\mathbf{F}^{*}\right) \rrbracket=0$.

Using the objectivity principle (2.4) and the residual stress free condition (2.5), we immediately obtain the expected result that every rotation $\mathbf{F}^{*}=\mathbf{R}^{*}$ is a trivial solution for (3.3) and (3.4). Of course, this is not a specific property of the composite under consideration but only a direct consequence of the objectivity principle together with (2.5).

To find non-trivial solutions for (3.3) and (3.4), we first establish their material counterparts. Under the homogeneous deformation field condition (3.1), the material stress tensor $\mathbf{S}^{(r)}$ of phase $r$ is related to its nominal one $\mathbf{P}^{(r)}$ by

$$
\mathbf{P}^{(r)}=\mathbf{F}^{*} \mathbf{S}^{(r)}
$$


By using (3.5) and invoking the objectivity principle condition (2.4), (3.2) and (3.3) can be equivalently rewritten as

$$
\begin{aligned}
& {\left[\mathbf{S}^{(2)}\left(\mathbf{E}^{*}\right)-\mathbf{S}^{(1)}\left(\mathbf{E}^{*}\right)\right] \mathbf{n}^{\perp}=\mathbf{0} \quad \text { on } \quad \Gamma,} \\
& \mathbf{S}^{(r) T}\left(\mathbf{E}^{*}\right)=\mathbf{S}^{(r)}\left(\mathbf{E}^{*}\right) \quad \text { over } \quad \Omega^{(r)},
\end{aligned}
$$

where $\mathbf{E}^{*}$ is the Green-Lagrange strain tensor associated to $\mathbf{F}^{*}$ by

$$
\mathbf{E}^{*}=\frac{1}{2}\left(\mathbf{C}^{*}-\mathbf{1}\right) \quad \text { with } \quad \mathbf{C}^{*}=\mathbf{F}^{* T} \mathbf{F}^{*} .
$$

Equation (3.6) must hold for any unit vector $\mathbf{n}^{\perp}$ normal to the fiber direction $\mathbf{n}$. As in the work of $\mathrm{He}$ [12], 1defining the fourth-order orthogonal projection tensors $\mathbb{N}$ and $\mathbb{N}^{\perp}$ by

$$
\mathbb{N}^{\perp}=1-\mathbb{N}=1-\mathbf{N} \otimes \mathbf{N},
$$

(3.6) can be written in a more convenient equivalent form:

$$
\mathbb{N}^{\perp} \llbracket \mathbf{S}\left(\mathbf{E}^{*}\right) \rrbracket=\mathbf{0} .
$$

While writing (3.10), the symmetric condition (3.7) has been tacitly employed. Bearing in mind the definition (3.9) of $\mathbb{N}^{\perp}$, we see that (3.10) is a system of five nonlinear equations with the six components of $\mathbf{E}^{*}$ as unknowns. Accounting for the residual-stress free hypothesis (2.10), it is immediate that $\mathbf{E}^{*}=\mathbf{0}$ is a trivial solution of (3.10). Next, following He [12], let us show that (3.10) admits other solutions than $\mathbf{E}^{*}=\mathbf{0}$, i.e., non-zero homogeneous strain fields can be produced in the composite.

First, the assumption that the nominal stress-strain relation $\mathbf{P}^{(r)}(\mathbf{E})$ of the matrix or fibers is continuously differentiable has the consequence that the material tangent tensors

$$
\mathbb{L}^{(r)}(\mathbf{E})=\nabla \mathbf{S}^{(r)}(\mathbf{E}) \quad(r=1,2)
$$

are continuous. Next, we introduce the jump of the material tangent tensor across $\Gamma$ as

$$
\llbracket \mathbb{L} \rrbracket=\mathbb{L}^{(2)}-\mathbb{L}^{(1)}
$$

and define the kernel space of $\mathbb{N}^{\perp} \llbracket \mathbf{L}(\mathbf{0}) \rrbracket$ by

$$
\operatorname{Ker}\left(\mathbb{N}^{\perp} \llbracket \mathbb{L}(\mathbf{0}) \rrbracket\right)=\left\{\delta \mathbf{E} \in \operatorname{Sym}: \mathbb{N}^{\perp} \llbracket \mathbb{L}(\mathbf{0}) \rrbracket \delta \mathbf{E}=\mathbf{0}\right\} .
$$

The dimension of this subspace of Sym is equal or greater than 1, i.e.,

$$
q=\operatorname{dim}\left(\operatorname{Ker}\left(\mathbb{N}^{\perp} \llbracket \mathbb{L}(\mathbf{0}) \rrbracket\right)\right) \geq 1
$$


because $\operatorname{dim}\left(\operatorname{Ker}\left(\mathbb{N}^{\perp}\right)\right)=1$ and $\operatorname{dim}(\operatorname{Ker}(\mathbb{A B}))=\max \{\operatorname{dim}(\operatorname{Ker}(\mathbb{A})), \operatorname{dim}(\operatorname{Ker}$ $(\mathbb{B}))\}$ for any two fourth-order tensors $\mathbb{A}$ and $\mathbb{B}$.

In view of the initial condition (2.10) and the fact (3.14), we can now apply the implicit function theorem of classical analysis (see, e.g., [3]) to infer the existence of a neighborhood $D$ of $\mathbf{E}^{*}=\mathbf{0}$, such that the solutions of Equation (3.10) within $D$ form a differentiable manifold $M$ of dimension $q$ :

$$
\operatorname{dim}(M)=q \geq 1
$$

with

$$
M=\left\{\mathbf{E}^{*} \in D \subseteq \operatorname{Sym}: \mathbb{N}^{\perp} \llbracket \mathbf{S}\left(\mathbf{E}^{*}\right) \rrbracket=\mathbf{0}\right\} .
$$

Thus, it follows from (3.15) to (3.16) that homogeneous elastic strains can be generated in the fiber-reinforced solid under consideration. The corresponding homogeneous elastic deformation fields are obtained by (3.1) with

$$
\mathbf{F}^{*}=\mathbf{R}^{*} \mathbf{U}^{*}=\mathbf{R}^{*}\left(2 \mathbf{E}^{*}+\mathbf{I}\right)^{1 / 2}, \quad \mathbf{R}^{*} \in S O(3), \quad \mathbf{E}^{*} \in M .
$$

For our purpose, it is useful to define another differentiable manifold:

$$
M^{\prime}=\left\{\mathbf{F}^{*}: \mathbf{F}^{*}=\mathbf{R}^{*}\left(2 \mathbf{E}^{*}+\mathbf{I}\right)^{1 / 2}, \mathbf{R}^{*} \in S O(3), \mathbf{E}^{*} \in M\right\} .
$$

We infer from (3.15) and (3.18) that

$$
\operatorname{dim}\left(M^{\prime}\right)=q \geq 4
$$

In fact, the manifold $M^{\prime}$ provides non-trivial solutions for (3.3) and (3.4).

For later use, let us introduce the plane $T_{M}\left(\mathbf{E}^{*}\right)$ tangent to $M$ at $\mathbf{E}^{*} \in M$ and the plane $T_{M^{\prime}}\left(\mathbf{F}^{*}\right)$ tangent to $M^{\prime}$ at $\mathbf{F}^{*} \in M^{\prime}$ :

$$
\begin{aligned}
& T_{M}\left(\mathbf{E}^{*}\right)=\operatorname{Ker}\left(\mathbb{N}^{\perp} \llbracket \mathbb{L}\left(\mathbf{E}^{*}\right) \rrbracket\right), \quad \mathbf{E}^{*} \in M, \\
& \left.T_{M^{\prime}}\left(\mathbf{F}^{*}\right)=\operatorname{Ker}(\mathbf{I} \underline{\otimes}(\mathbf{I}-\mathbf{N})) \llbracket \mathbb{K}\left(\mathbf{F}^{*}\right) \rrbracket\right), \quad \mathbf{F}^{*} \in M^{\prime} .
\end{aligned}
$$

In (3.21), $\llbracket \mathbb{K}\left(\mathbf{F}^{*}\right) \rrbracket$ is the jump of the phase nominal tangent tensors across the interface $\Gamma$, i.e.,

$$
\llbracket \mathbb{K} \rrbracket=\mathbb{K}^{(2)}-\mathbb{K}^{(1)}
$$

with

$$
\mathbb{K}^{(r)}=\nabla \mathbf{P}^{(r)} \quad(r=1,2),
$$


and the kernel space of $(\mathbf{I} \underline{\otimes}(\mathbf{I}-\mathbf{N})) \llbracket \mathbb{K}\left(\mathbf{F}^{*}\right) \rrbracket$ is defined as

$$
\operatorname{Ker}\left((\mathbf{I} \underline{\otimes}(\mathbf{I}-\mathbf{N})) \llbracket \mathbb{K}\left(\mathbf{F}^{*}\right) \rrbracket\right)=\left\{\delta \mathbf{F} \in \operatorname{Lin}:(\mathbf{I} \underline{\otimes}(\mathbf{I}-\mathbf{N})) \llbracket \mathbb{K}\left(\mathbf{F}^{*}\right) \rrbracket \delta \mathbf{F}=\mathbf{0}\right\} .
$$

In passing and for later use, observe that the basic formula (3.5) gives rise o the following relation between the nominal and material tangent tensors (see, e.g., $[4,6])$ :

$$
\mathbb{K}^{(r)}\left(\mathbf{F}^{*}\right)=\underline{\mathbf{I}} \underline{\otimes} \mathbf{S}^{(r)}\left(\mathbf{E}^{*}\right)+\left(\mathbf{F}^{*} \underline{\otimes} \mathbf{I}\right) \mathbb{L}^{(r)}\left(\mathbf{E}^{*}\right)\left(\mathbf{F}^{* T} \underline{\otimes} \mathbf{I}\right) .
$$

Lastly, it is directly deduced from (3.15) and (3.19) that

$$
\operatorname{dim}\left(T_{M}\left(\mathbf{E}^{*}\right)\right)=q \geq 1, \quad \operatorname{dim}\left(T_{M^{\prime}}\left(\mathbf{F}^{*}\right)\right)=q \geq 4
$$

provided $\mathbf{E}^{*} \in M$ and $\mathbf{F}^{*} \in M^{\prime}$.

\section{Microstructure-Independent Exact Results}

The possibility of generating non-trivial homogeneous elastic deformations in the fiber-reinforced solid has at least two important consequences: (i) The effective nominal or material stress-strain relation can be explicitly and exactly determined for every $\mathbf{F}^{*} \in M^{\prime}$; (ii) exact connections exist between the effective elastic nominal (or material) tangent moduli evaluated at any $\mathbf{F}^{*} \in M^{\prime}$ (or $\mathbf{E}^{*} \in$ $M$ ). This section has the purpose of establishing these results which turn out to be independent of the transverse geometry and distribution of the fibers.

\subsection{EXACT RESULTS FOR THE EFFECTIVE NOMINAL AND MATERIAL STRESS-STRAIN RELATIONS}

For any surface loading specified by (2.16) with $\overline{\mathbf{F}}=\mathbf{F}^{*} \in M^{\prime}$, the resulting deformation field is homogeneous over $\Omega$. Correspondingly, the nominal stress field $\mathbf{P}\left(\mathbf{x}, \mathbf{F}^{*}\right)$ calculated by (2.1) is homogenous in each phase. Further, owing to the satisfaction of (3.4), the part $[\underline{\mathbf{I}} \otimes(\mathbf{I}-\mathbf{N})] \mathbf{P}\left(\mathbf{x}, \mathbf{F}^{*}\right)$ of $\mathbf{P}\left(\mathbf{x}, \mathbf{F}^{*}\right)$ is homogeneous over $\Omega$. So, using (2.12), we can exactly determine the effective nominal stressstrain relation (2.15) for any $\overline{\mathbf{F}}=\mathbf{F}^{*} \in M^{\prime}$ as follows:

$$
\overline{\mathbf{P}}=\hat{\mathbf{P}}\left(\mathbf{F}^{*}\right)=<\mathbf{P}\left(\mathbf{x}, \mathbf{F}^{*}\right)>=c^{(1)} \mathbf{P}^{(1)}\left(\mathbf{F}^{*}\right)+c^{(2)} \mathbf{P}^{(2)}\left(\mathbf{F}^{*}\right) .
$$

Further, recalling that $c^{(r)}$ denotes the volume fraction of phase $r,(4.1)$ can be split into

$$
(\underline{\mathbf{I}} \underline{\mathbf{N}}) \hat{\mathbf{P}}\left(\mathbf{F}^{*}\right)=(\underline{\mathbf{I} \otimes} \mathbf{N})\left[c^{(1)} \mathbf{P}^{(1)}\left(\mathbf{F}^{*}\right)+c^{(2)} \mathbf{P}^{(2)}\left(\mathbf{F}^{*}\right)\right],
$$




$$
\left.[\underline{\mathbf{I} \otimes}(\mathbf{I}-\mathbf{N})] \hat{\mathbf{P}}\left(\mathbf{F}^{*}\right)=[\underline{\mathbf{I} \otimes}(\mathbf{I}-\mathbf{N})] \mathbf{P}^{(1)}\left(\mathbf{F}^{*}\right)=[\underline{\mathbf{I} \otimes}(\mathbf{I}-\mathbf{N})] \mathbf{P}^{(2)}\left(\mathbf{F}^{*}\right)\right] .
$$

Using (2.14) and (4.1), we obtain the associated effective material stress:

$$
\overline{\mathbf{S}}=\mathbf{F}^{*-1} \hat{\mathbf{P}}\left(\mathbf{F}^{*}\right)=c^{(1)} \mathbf{F}^{*-1} \mathbf{P}^{(1)}\left(\mathbf{F}^{*}\right)+c^{(2)} \mathbf{F}^{*-1} \mathbf{P}^{(2)}\left(\mathbf{F}^{*}\right) .
$$

Since $\mathbf{P}^{(r)}\left(\mathbf{F}^{*}\right)$ verifies the principle of objectivity as specified by (2.4), we can invoke (2.8) and (2.14) to put (4.4) in a simpler equivalent form:

$$
\overline{\mathbf{S}}=\hat{\mathbf{S}}\left(\mathbf{E}^{*}\right)=c^{(1)} \mathbf{S}^{(1)}\left(\mathbf{E}^{*}\right)+c^{(2)} \mathbf{S}^{(2)}\left(\mathbf{E}^{*}\right),
$$

where $\hat{\mathbf{S}}\left(\mathbf{E}^{*}\right)=\mathbf{F}^{*-1} \hat{\mathbf{P}}\left(\mathbf{F}^{*}\right)$ is the effective material stress-strain relation $\hat{\mathbf{S}}(\mathbf{E})$ evaluated at $\overline{\mathbf{E}}=\mathbf{E}^{*} \in M$.

Interestingly, we observe that (4.5) amounts to $\overline{\mathbf{S}}=<\mathbf{S}\left(\mathbf{x}, \mathbf{E}^{*}\right)>$. This seems to be in contradiction with the general fact that $\overline{\mathbf{E}} \neq<\mathbf{E}>$ and $\overline{\mathbf{S}} \neq<\mathbf{S}>$ recalled just after equation (2.14). In reality, in the special case where a homogeneous deformation field $\mathbf{y}(\mathbf{x})=\mathbf{F}^{*} \mathbf{x}$ over $\Omega$ represents a solution for the homogenization problem, it results from (2.13) to (2.14) that $\overline{\mathbf{E}}=\langle\mathbf{E}\rangle=\mathbf{E}^{*}$ and $\overline{\mathbf{S}}=<\mathbf{S}>=<\mathbf{S}\left(\mathbf{x}, \mathbf{E}^{*}\right)>$.

When the phases of the composite are hyperelastic, i.e. the nominal stressstrain relation $\mathbf{P}^{(r)}(\mathbf{F})$ of phase $r$ in (2.1) is given by

$$
\mathbf{P}^{(r)}(\mathbf{F})=\frac{\partial w^{(r)}(\mathbf{F})}{\partial \mathbf{F}}
$$

where $w^{(r)}(\mathbf{F})$ is the strain-energy function of phase $r$, then the existence of homogeneous deformation fields implies that the effective strain-energy function $\hat{w}(\overline{\mathbf{F}})$ can be exactly evaluated for all $\mathbf{F}^{*} \in M^{\prime}$ as follows:

$$
\hat{w}\left(\mathbf{F}^{*}\right)=c^{(1)} w^{(1)}\left(\mathbf{F}^{*}\right)+c^{(2)} w^{(2)}\left(\mathbf{F}^{*}\right) .
$$

Thus, the Voigt bound established by Ogden [28] in the case of finite elasticity is achieved by fiber-reinforced composites whenever $\mathbf{F}^{*} \in M^{\prime}$.

\subsection{EXACT CONNECTIONS BETWEEN EFFECTIVE NOMINAL TANGENT MODULI}

By definition, the effective nominal tangent tensor $\mathbb{A}(\overline{\mathbf{F}})$ associated to (2.15) is given by

$$
\mathbb{A}(\overline{\mathbf{F}})=\nabla \hat{\mathbf{P}}(\overline{\mathbf{F}})
$$

Now, let us show that exact connections exist between the components of $\mathbb{A}\left(\mathbf{F}^{*}\right)$ with $\mathbf{F}^{*} \in M^{\prime}$. 
In view of (3.21) and (3.26) $)_{2}$, it makes sense to introduce the orthogonal projection operator $\mathbb{H}\left(\mathbf{F}^{*}\right)$ from Lin to $T_{M^{\prime}}\left(\mathbf{F}^{*}\right)$ through

$$
\operatorname{Ker}\left((\mathbf{I} \underline{\otimes}(\mathbf{I}-\mathbf{N})) \llbracket \mathbb{K}\left(\mathbf{F}^{*}\right) \rrbracket\right)=\left\{\delta \mathbf{F}^{*}: \delta \mathbf{F}^{*}=\left(\mathbb{H}\left(\mathbf{F}^{*}\right)\right) \delta \mathbf{F}, \delta \mathbf{F} \in \operatorname{Lin}\right\}
$$

and the complementary orthogonal projection operator $\mathbb{H}^{\perp}\left(\mathbf{F}^{*}\right)$ by

$$
\mathbb{H}^{\perp}\left(\mathbf{F}^{*}\right)=\mathbb{I}-\mathbb{H}\left(\mathbf{F}^{*}\right) .
$$

In particular, when $\operatorname{det}\left(\llbracket \mathbb{K}\left(\mathbf{F}^{*}\right) \rrbracket\right) \neq 0$, we have $\mathbb{H}\left(\mathbf{F}^{*}\right)=\mathbf{I} \otimes(\mathbf{I}-\mathbf{N})$ and $\mathbb{H}^{\perp}$ $\left(\mathbf{F}^{*}\right)=\mathbf{I} \otimes \mathbf{N}$. In what follows, it should be kept in mind that $\overline{\mathbb{H}}\left(\mathbf{F}^{*}\right)$ and $\mathbb{H}^{\perp}\left(\mathbf{F}^{*}\right)$ are entirely determined by $(\mathbf{I} \otimes(\mathbf{I}-\mathbf{N})) \llbracket \mathbb{K}\left(\mathbf{F}^{*}\right) \rrbracket$. With the aid of $\mathbb{H}\left(\mathbf{F}^{*}\right)$ and $\mathbb{H}^{\perp}\left(\mathbf{F}^{*}\right)$, every variation $\delta \mathbf{F}$ of a local deformation gradient field $\mathbf{F}^{*} \in M^{\prime}$ admits the following decomposition:

$$
\delta \mathbf{F}=\delta \mathbf{F}^{*}+\delta \mathbf{F}^{\prime}, \quad \delta \mathbf{F}^{*}=\mathbb{H} \delta \mathbf{F}, \quad \delta \mathbf{F}^{\prime}=\mathbb{H}^{\perp} \delta \mathbf{F} .
$$

In other words, each variation $\delta \mathbf{F}$ of $\mathbf{F}^{*} \in M^{\prime}$ can be uniquely decomposed into one component $\delta \mathbf{F}^{*}$ tangent to $M^{\prime}$ at $\mathbf{F}^{*}$ and one component $\delta \mathbf{F}^{\prime}$ perpendicular to the tangent plane $T_{M^{\prime}}\left(\mathbf{F}^{*}\right)$. Remark that $\delta \mathbf{F}^{*}$ induces a homogeneous deformation field in the fiber-reinforced composite. So, for any variation $\delta \overline{\mathbf{F}}$ of a macroscopic deformation gradient $\overline{\mathbf{F}}=\mathbf{F}^{*} \in M^{\prime}$, the decomposition

$$
\delta \overline{\mathbf{F}}=\delta \mathbf{F}^{*}+\delta \overline{\mathbf{F}}^{\prime}, \quad \delta \mathbf{F}^{*}=\mathbb{H} \delta \overline{\mathbf{F}}, \quad \delta \overline{\mathbf{F}}^{\prime}=\mathbb{H}^{\perp} \delta \overline{\mathbf{F}}
$$

holds as well.

The phase nominal incremental stress-strain relations associated to (2.1) and the effective nominal incremental stress-strain relation corresponding to (2.15) take the following respective forms:

$$
\begin{aligned}
& \delta \mathbf{P}=\delta \mathbf{P}(\mathbf{X}, \mathbf{F}, \delta \mathbf{F})=\sum_{r=1}^{r=2} \chi^{(r)}(\mathbf{x}) \delta \mathbf{P}^{(r)}(\mathbf{F}, \delta \mathbf{F})=\sum_{r=1}^{r=2} \chi^{(r)}(\mathbf{x}) \mathbb{K}^{(r)}(\mathbf{F}) \delta \mathbf{F} \\
& \delta \overline{\mathbf{P}}=\delta \hat{\mathbf{P}}(\overline{\mathbf{F}}, \delta \overline{\mathbf{F}})=(\nabla \hat{\mathbf{P}}(\overline{\mathbf{F}})) \delta \overline{\mathbf{F}}=\mathbb{A}(\overline{\mathbf{F}}) \delta \overline{\mathbf{F}} .
\end{aligned}
$$

When $\mathbf{F}^{*} \in M^{\prime}$, then $\overline{\mathbf{F}}=\mathbf{F}^{*}$ and use of the orthogonal projection operator $\mathbb{H}\left(\mathbf{F}^{*}\right)$ and $\mathbb{H}^{\perp}\left(\mathbf{F}^{*}\right)$ allows to split (4.13) into

$$
\begin{aligned}
& \mathbb{H} \delta \mathbf{P}=\mathbb{H}\left(\sum_{r=1}^{r=2} \chi^{(r)}(\mathbf{x}) \mathbb{K}^{(r)}\right) \mathbb{H} \delta \mathbf{F}+\mathbb{H}\left(\sum_{r=1}^{r=2} \chi^{(r)}(\mathbf{x}) \mathbb{K}^{(r)}\right) \mathbb{H}^{\perp} \delta \mathbf{F}, \\
& \mathbb{H}^{\perp} \delta \mathbf{P}=\mathbb{H}^{\perp}\left(\sum_{r=1}^{r=2} \chi^{(r)}(\mathbf{x}) \mathbb{K}^{(r)}\right) \mathbb{H} \delta \mathbf{F}+\mathbb{H}^{\perp}\left(\sum_{r=1}^{r=2} \chi^{(r)}(\mathbf{x}) \mathbb{K}^{(r)}\right) \mathbb{H}^{\perp} \delta \mathbf{F},
\end{aligned}
$$


and (4.14) into

$$
\begin{aligned}
& \mathbb{H} \delta \overline{\mathbf{P}}=\mathbb{H} \mathbb{A} \mathbb{H} \delta \overline{\mathbf{F}}+\mathbb{H} \mathbb{A} \mathbb{H}^{\perp} \delta \overline{\mathbf{F}}, \\
& \mathbb{H}^{\perp} \delta \overline{\mathbf{P}}=\mathbb{H}^{\perp} \mathbb{A} \mathbb{H} \delta \overline{\mathbf{F}}+\mathbb{H}^{\perp} \mathbb{A} \mathbb{H}^{\perp} \delta \overline{\mathbf{F}} .
\end{aligned}
$$

In (4.15)-(4.18) and in what follows, for notational simplicity the dependence on $\mathbf{F}^{*} \in M^{\prime}$ is dropped as no confusion is possible.

The volume average of (4.15) and the one of (4.16) give

$$
\begin{aligned}
& \mathbb{H} \delta \overline{\mathbf{P}}=\sum_{r=1}^{r=2} c^{(r)}\left(\mathbb{H} \mathbb{K}^{(r)} \mathbb{H}\right) \delta \mathbf{F}^{*}+\sum_{r=1}^{r=2} c^{(r)}\left(\mathbb{H} \mathbb{K}^{(r)} \mathbb{H}^{\perp}\right)<\delta \mathbf{F}^{\prime}>_{r}, \\
& \mathbb{H}^{\perp} \delta \overline{\mathbf{P}}=\sum_{r=1}^{r=2} c^{(r)}\left(\mathbb{H}^{\perp} \mathbb{K}^{(r)} \mathbb{H}\right) \delta \mathbf{F}^{*}+\sum_{r=1}^{r=2} c^{(r)}\left(\mathbb{H}^{\perp} \mathbb{K}^{(r)} \mathbb{H}^{\perp}\right)<\delta \mathbf{F}^{\prime}>_{r}
\end{aligned}
$$

Using $\delta \overline{\mathbf{F}}^{\prime}=c^{(1)}<\delta \mathbf{F}^{\prime}>_{1}+c^{(2)}<\delta \mathbf{F}^{\prime}>_{2}$ and comparing the above two expressions with (4.17) and (4.18), we derive

$$
\begin{gathered}
\mathbb{H}(\mathbb{A}-<\mathbb{K}>) \mathbb{H} \delta \mathbf{F}^{*}+\mathbb{H}\left(\mathbb{A}-\mathbb{K}^{(1)}\right) \mathbb{H}^{\perp} \delta \overline{\mathbf{F}}^{\prime}=c^{(2)} \mathbb{H} \llbracket \mathbb{K} \rrbracket \mathbb{H} \perp^{\perp}<\delta \mathbf{F}^{\prime}>_{2}, \\
\mathbb{H}^{\perp}(\mathbb{A}-<\mathbb{K}>) \mathbb{H} \delta \mathbf{F}^{*}+\mathbb{H}^{\perp}\left(\mathbb{A}-\mathbb{K}^{(1)}\right) \mathbb{H}^{\perp} \delta \mathbf{F}^{\prime}=c^{(2)} \mathbb{H}^{\perp} \llbracket \mathbb{K} \rrbracket \mathbb{H}^{\perp}<\delta \mathbf{F}^{\prime}>_{2},
\end{gathered}
$$

where $<\mathbb{K}>=c^{(1)} \mathbb{K}^{(1)}+c^{(2)} \mathbb{K}^{(2)}$. To obtain a set of exact connections between the components of $\mathbb{A}$, we first pose $\delta \overline{\mathbf{F}}^{\prime}=0$, eliminate $\left\langle\delta \mathbf{F}^{\prime}\right\rangle_{2}$ and prescribe $\delta \mathbf{F}^{*}$ arbitrarily in (4.21) and (4.22). This gives

$$
\mathbb{H}\left[\left(\llbracket \mathbb{K} \rrbracket\left(\mathbb{H}^{\perp} \llbracket \mathbb{K} \rrbracket \mathbb{H}^{\perp}\right)^{-1}-\mathbb{I}\right)(\mathbb{A}-<\mathbb{K}>)\right] \mathbb{H}=0 .
$$

Next, setting $\delta \mathbf{F}^{*}=0$, eliminating $\left\langle\delta \mathbf{F}^{\prime}\right\rangle_{2}$ and letting $\delta \overline{\mathbf{F}}^{\prime}$ be arbitrary in (4.21) and (4.22), yields

$$
\mathbb{H}\left[\left(\llbracket \mathbb{K} \rrbracket\left(\mathbb{H}^{\perp} \llbracket \mathbb{K} \rrbracket \mathbb{H}^{\perp}\right)^{-1}-\mathbb{I}\right)\left(\mathbb{A}-\mathbb{K}^{(1)}\right)\right] \mathbb{H}^{\perp}=0 .
$$

In (4.23) and (4.24), the partial inverse $\left(\mathbb{H}^{\perp} \llbracket \mathbb{K} \rrbracket \mathbb{H}^{\perp}\right)^{-1}$ should be understood in the sense that $\left(\mathbb{H}^{\perp} \llbracket \mathbb{K} \rrbracket \mathbb{H}^{\perp}\right)^{-1}\left(\mathbb{H}^{\perp} \llbracket \mathbb{K} \rrbracket \mathbb{H}^{\perp}\right)=\left(\mathbb{H}^{\perp} \llbracket \mathbb{K} \rrbracket \mathbb{H}{ }^{\perp}\right) \quad\left(\mathbb{H}^{\perp} \llbracket \mathbb{K} \rrbracket \mathbb{H}^{\perp}\right)^{-1}=$ $\mathbb{H}^{\perp}$.

The exact relations (4.23) and (4.24) establish connections between the effective nominal tangent moduli evaluated at each point $\mathbf{F}^{*} \in M^{\prime}$. These 
connections are microstructure-independent in the sense that they hold irrespectively of the transverse geometry and distribution of the fibers. They have the direct consequence that the number of independent effective nominal tangent moduli evaluated for all point $\mathbf{F}^{*} \in M^{\prime}$ is reduced. The degree of reduction is determined by the dimension of the kernel space of $(\mathbf{I} \otimes(\mathbf{I}-$ $\mathbf{N})) \llbracket \mathbb{K}\left(\mathbf{F}^{*}\right) \rrbracket$ as defined by (4.9).

\subsection{EXACT CONNECTIONS BETWEEN EFFECTIVE MATERIAL TANGENT MODULI}

By definition, the effective material tangent tensor $\mathbb{B}(\overline{\mathbf{E}})$ associated to the effective material stress-strain relation $\hat{\mathbf{S}}(\mathbf{E})$ is provided by

$$
\mathbb{B}(\overline{\mathbf{E}})=\nabla \hat{\mathbf{S}}(\overline{\mathbf{E}})
$$

The fact that uniform strains can be produced in the composite and constitute a differential manifold $M$ specified by (3.16) gives rise to exact connections between the components of $\mathbb{B}\left(\mathbf{E}^{*}\right)$ with $\mathbf{E}^{*} \in M$. However, the method used in the last paragraph to establish connections between the components of the effective nominal tangent tensor $\mathbb{A}\left(\mathbf{F}^{*}\right)$ with $\mathbf{F}^{*} \in M^{\prime}$ cannot be applied here, because $\delta \overline{\mathbf{E}} \neq<\delta \mathbf{E}>$ and $\delta \overline{\mathbf{S}} \neq<\delta \mathbf{S}>$. Instead, an efficient way to achieve the purpose consists in starting from the established nominal connections (4.23) and (4.24) and using the following relation

$$
\mathbb{A}\left(\mathbf{F}^{*}\right)=\underline{\mathbf{I}} \underline{\hat{\mathbf{S}}}\left(\mathbf{E}^{*}\right)+\left(\mathbf{F}^{*} \underline{\otimes} \mathbf{I}\right) \mathbb{B}\left(\mathbf{E}^{*}\right)\left(\mathbf{F}^{* T} \underline{\otimes} \mathbf{I}\right)
$$

together with the corresponding phase relation (3.25).

Introducing (4.26) and (3.25) into (4.23) and (4.24), we obtain

$$
\begin{aligned}
& \mathbb{H}\left[\left(\mathbf{I} \underline{\otimes} \llbracket \mathbf{S} \rrbracket+\left(\mathbf{F}^{*} \underline{\otimes} \mathbf{I}\right) \llbracket \mathbb{L} \rrbracket\left(\mathbf{F}^{* T} \underline{\otimes} \mathbf{I}\right)\right)\left(\mathbb{H}^{\perp}\left(\mathbf{I} \underline{\otimes} \llbracket \mathbf{S} \rrbracket+\left(\mathbf{F}^{*} \underline{\otimes} \mathbf{I}\right) \llbracket \mathbb{L} \rrbracket\left(\mathbf{F}^{* T} \underline{\otimes} \mathbf{I}\right)\right) \mathbb{H}^{\perp}\right)^{-1}-\mathbb{I}\right) \\
& \left.\times\left(\underline{\mathbf{I}} \underline{\otimes}(\hat{\mathbf{S}}-<\mathbf{S}>)+\left(\mathbf{F}^{*} \underline{\otimes} \mathbf{I}\right)(\mathbb{B}-<\mathbb{L}>)\left(\mathbf{F}^{* T} \underline{\otimes} \mathbf{I}\right)\right)\right] \mathbb{H}=0, \\
& \mathbb{H}\left[\left(\mathbf{I} \otimes \underline{\mathbf{S}} \rrbracket+\left(\mathbf{F}^{*} \underline{\otimes} \mathbf{I}\right) \llbracket \mathbb{L} \rrbracket\left(\mathbf{F}^{* T} \underline{\otimes} \mathbf{I}\right)\left(\mathbb{H}^{\perp}\left(\underline{\mathbf{I}} \underline{\otimes} \llbracket \mathbf{S} \rrbracket+\left(\mathbf{F}^{*} \underline{\otimes} \mathbf{I}\right) \llbracket \mathbb{L} \rrbracket\left(\mathbf{F}^{* T} \underline{\otimes} \mathbf{I}\right)\right) \mathbb{H}^{\perp}\right)^{-1}-\mathbb{I}\right)\right. \\
& \left.\times\left(\underline{\mathbf{I}} \underline{\otimes}\left(\hat{\mathbf{S}}-\mathbf{S}^{(1)}\right)+\left(\mathbf{F}^{*} \underline{\otimes} \mathbf{I}\right)\left(\mathbb{B}-\mathbb{L}^{(1)}\right)\left(\mathbf{F}^{* T} \underline{\otimes} \mathbf{I}\right)\right)\right] \mathbb{H}^{\perp}=0 \text {. }
\end{aligned}
$$

These exact connections are much more complex than (4.23) and (4.24). However, in the particular case where $\mathbf{F}^{*}=\mathbf{I}$ and $\mathbf{E}^{*}=\mathbf{0}$, the residual-stress free condition (2.10) means that $\mathbf{S}^{(r)}(\mathbf{0})=\mathbf{0}$ and implies that $\hat{\mathbf{S}}(\mathbf{0})=\mathbf{0}$. Correspondingly, (4.27) and (4.28) can be considerably simplified into

$$
\mathbb{H}_{0}\left[\left(\llbracket \mathbb{L}_{0} \rrbracket\left(\mathbb{H}_{0}^{\perp} \llbracket \mathbb{L}_{0} \rrbracket \mathbb{H}_{0}^{\perp}\right)^{-1}-\mathbb{I}\right)\left(\mathbb{B}_{0}-<\mathbb{L}_{0}>\right)\right] \mathbb{H}_{0}=0
$$




$$
\mathbb{H}_{0}\left[\left(\llbracket \mathbb{L}_{0} \rrbracket\left(\mathbb{H}_{0}^{\perp} \llbracket \mathbb{L}_{0} \rrbracket \mathbb{H}_{0}^{\perp}\right)^{-1}-\mathbb{I}\right)\left(\mathbb{B}_{0}-\mathbb{L}_{0}^{(1)}\right)\right] \mathbb{H}_{0}^{\perp}=\mathbb{0}
$$

where a quantity with the subscript 0 signifies that it is evaluated at $\overline{\mathbf{E}}=\mathbf{E}^{*}=\mathbf{0}$ or $\mathbf{F}^{*}=\mathbf{I}$.

In the case of hyperelastic infinitesimal strains and under the condition that $\mathbb{L}_{0}^{(2)}-\mathbb{L}_{0}^{(1)}$ is not singular, i.e., $\operatorname{det}\left(\mathbb{L}_{0}^{(2)}-\mathbb{L}_{0}^{(1)}\right) \neq 0$, the connections (4.29) and (4.30) reduce to those given by Dvorak [7] for linear elasticity.

\section{Example}

The general results given in the previous sections are now illustrated by considering a simple but physically meaningful example where phase 1 is a compressible Mooney-Rivlin material and phase 2 consists of unidirectional cylindrical pores.

The matrix phase of the porous material under consideration is characterized by the Mooney-Rivlin strain-energy function (see, e.g., $[5,16])$ :

$$
\begin{aligned}
w^{(1)}(\mathbf{F})=\widetilde{w}^{(1)}\left(J_{i}\right)= & \alpha_{1}^{(1)}\left(J_{3}^{1 / 2}-1\right)^{2}-\left(\alpha_{2}^{(1)}+2 \alpha_{3}^{(1)}\right) \ln J_{3} \\
& +\alpha_{2}^{(1)}\left(J_{1}-3\right)+\alpha_{3}^{(1)}\left(J_{2}-3\right),
\end{aligned}
$$

where $\alpha_{i}^{(1)} \geq 0$ are the material parameters of the matrix phase and $J_{i}$ are the principal invariants of the right Cauchy-Green strain tensor $\mathbf{C}=\mathbf{F}^{T} \mathbf{F}$ defined by

$$
J_{1}=\operatorname{tr} \mathbf{C}, \quad J_{2}=\frac{1}{2}\left[(\operatorname{tr} \mathbf{C})^{2}-\operatorname{tr}\left(\mathbf{C}^{2}\right)\right], \quad J_{3}=\operatorname{det} \mathbf{C} .
$$

Clearly, for phase 2 made of cylindrical pores, we have $w^{(2)}(\mathbf{F})=0$. Applying the classical formula (see, e.g., [29])

$$
\mathbf{S}^{(1)}=2 \frac{\partial \widetilde{w}^{(1)}}{\partial \mathbf{C}}=2\left[\left(\frac{\partial \widetilde{w}^{(1)}}{\partial J_{1}}+J_{1} \frac{\partial \widetilde{w}^{(1)}}{\partial J_{2}}\right) \mathbf{I}-\frac{\partial \widetilde{w}^{(1)}}{\partial J_{2}} \mathbf{C}+J_{3} \frac{\partial \widetilde{w}^{(1)}}{\partial J_{3}} \mathbf{C}^{-1}\right]
$$

to (5.1) gives the material stress of the matrix phase

$$
\mathbf{S}^{(1)}=2\left(\alpha_{2}^{(1)}+\alpha_{3}^{(1)} J_{1}\right) \mathbf{I}-2 \alpha_{3}^{(1)} \mathbf{C}+2\left[\alpha_{1}^{(1)}\left(J_{3}-J_{3}^{1 / 2}\right)-\alpha_{2}^{(1)}-2 \alpha_{3}^{(1)}\right] \mathbf{C}^{-1} .
$$


Next, the expression for the corresponding nominal stress is obtained as follows:

$$
\begin{aligned}
\mathbf{P}^{(1)}=\mathbf{F S}^{(1)}= & 2\left(\alpha_{2}^{(1)}+\alpha_{3}^{(1)} J_{1}\right) \mathbf{F}-2 \alpha_{3}^{(1)} \mathbf{F C} \\
& +2\left[\alpha_{1}^{(1)}\left(J_{3}-J_{3}^{1 / 2}\right)-\alpha_{2}^{(1)}-2 \alpha_{3}^{(1)}\right] \mathbf{F}^{-T} .
\end{aligned}
$$

Regarding phase 2, we have $\mathbf{S}^{(2)}=\mathbf{P}^{(2)}=\mathbf{0}$.

As shown in Section 3, finding homogeneous material strain fields amounts to solving the system (3.10) of nonlinear equations. To this end, let us introduce an orthonormal basis $\left\{\mathbf{e}_{1}, \mathbf{e}_{1}, \mathbf{e}_{3}\right\}$ with the pore direction $\mathbf{n}$ coinciding with $\mathbf{e}_{1}$. Then, substituting (5.4) into the system of equations $\mathbb{N}^{\perp} \llbracket \mathbf{S}(\mathbf{E}) \rrbracket \mathbf{C}=\mathbf{0}$ which is equivalent to (3.10), it follows that

$$
\mathbb{N}^{\perp}\left\{\left(\alpha_{2}+\alpha_{3} J_{1}\right) \mathbf{C}-\alpha_{3} \mathbf{C}^{2}+\left[\alpha_{1}\left(J_{3}-J_{3}^{1 / 2}\right)-\alpha_{2}-2 \alpha_{3}\right] \mathbf{I}\right\}=\mathbf{0},
$$

where $\mathbb{N}^{\perp}=1-\mathbf{e}_{1} \otimes \mathbf{e}_{1} \otimes \mathbf{e}_{1} \otimes \mathbf{e}_{1}$. In (5.6) and in what follows, we set $\alpha_{i}=\alpha_{i}^{(1)}$ $(i=1,2,3)$ for notational simplicity.

To find all the solutions for the system of nonlinear equation (5.6), we first observe the fact that, given any right Cauchy-Green strain tensor $\mathbf{C}$, an orthonormal basis $\left\{\mathbf{e}_{1}, \mathbf{e}_{2}, \mathbf{e}_{3}\right\}$ can always be chosen such that $\mathbf{e}_{1}$ corresponds to the direction $\mathbf{n}$ of cylindrical pores and the matrix of $\mathbf{C}$ relative to $\left\{\mathbf{e}_{1}, \mathbf{e}_{2}, \mathbf{e}_{3}\right\}$ takes the form

$$
\mathbf{C}=\left[\begin{array}{ccc}
C_{11} & C_{12} & C_{13} \\
C_{12} & C_{22} & 0 \\
C_{13} & 0 & C_{33}
\end{array}\right] .
$$

In fact, it is always possible to rotate the transverse-plane orthonormal basis $\left\{\mathbf{e}_{2}\right.$, $\left.\mathbf{e}_{3}\right\}$ about the axis $\mathbf{e}_{1}$ so that $C_{23}=C_{32}=0$. Correspondingly,

$$
\begin{aligned}
& J_{1}=C_{11}+C_{22}+C_{33}, \\
& J_{2}=C_{11} C_{22}+C_{22} C_{33}+C_{33} C_{11}-C_{12}^{2}-C_{13}^{2}, \\
& J_{3}=C_{11} C_{22} C_{33}-C_{12}^{2} C_{33}-C_{13}^{2} C_{22} .
\end{aligned}
$$

Introducing (5.7) and (5.8a, 5.8b, 5.8c) into (5.6), we obtain five nonlinear equations:

$$
\begin{aligned}
\alpha_{2} C_{12}+\alpha_{3} C_{33} C_{12} & =0, \\
\alpha_{2} C_{13}+\alpha_{3} C_{22} C_{13} & =0, \\
\alpha_{3} C_{12} C_{13} & =0,
\end{aligned}
$$




$$
\begin{array}{r}
\alpha_{1}\left(C_{11} C_{22} C_{33}-C_{12}^{2} C_{33}-C_{13}^{2} C_{22}-\sqrt{C_{11} C_{22} C_{33}-C_{12}^{2} C_{33}-C_{13}^{2} C_{22}}\right) \\
+\alpha_{2} C_{22}+\alpha_{3}\left(C_{11} C_{22}+C_{33} C_{22}-C_{12}^{2}\right)-\alpha_{2}-2 \alpha_{3}=0 \\
\alpha_{1}\left(C_{11} C_{22} C_{33}-C_{12}^{2} C_{33}-C_{13}^{2} C_{22}-\sqrt{C_{11} C_{22} C_{33}-C_{12}^{2} C_{33}-C_{13}^{2} C_{22}}\right) \\
+\alpha_{2} C_{33}+\alpha_{3}\left(C_{11} C_{33}+C_{22} C_{33}-C_{13}^{2}\right)-\alpha_{2}-2 \alpha_{3}=0 .
\end{array}
$$

In Appendix $\mathrm{A}$, it is proved that, due to the requirement that $\mathbf{C}$ be positive definite, quations (5.9)-(5.13) admit only the solution expressed by

$$
\mathbf{C}^{*}=\mathbf{U}^{* 2}=\lambda_{1}^{2} \mathbf{e}_{1} \otimes \mathbf{e}_{1}+\lambda_{2}^{2}\left(\mathbf{I}-\mathbf{e}_{1} \otimes \mathbf{e}_{1}\right)
$$

with

$$
\lambda_{1}(t)=\frac{\alpha_{1} t+\sqrt{\alpha_{1}^{2} t^{2}-4\left(\alpha_{3}+\alpha_{1} t^{2}\right)\left[\alpha_{3}\left(t^{4}-2\right)+\alpha_{2}\left(t^{2}-1\right)\right]}}{2\left(\alpha_{3}+\alpha_{1} t^{2}\right) t}, \quad \lambda_{2}(t)=t
$$

where $t \in] 0,+\infty$ [ is a positive parameter. The tensors $\mathbf{E}^{*}=\left(\mathbf{C}^{*}-\mathbf{I}\right) / 2$ with $\mathbf{C}^{*}$ specified by (5.14) and (5.15) are transversely isotropic with respect to $\mathbf{e}_{1}$ and form a manifold $M$ of dimension $q=1$ in the space Sym. Furthermore, using (3.18), we obtain the manifold

$$
M^{\prime}=\left\{\mathbf{F}^{*}: \mathbf{F}^{*}=\mathbf{R}^{*}(2 \mathbf{E}+\mathbf{I})^{1 / 2}, \mathbf{R}^{*} \in S O(3), \mathbf{E}^{*} \in M\right\}
$$

in the space Lin. Then, all admissible homogeneous deformation fields for the porous material under consideration are given by (3.1) with $\mathbf{F}^{*} \in M^{\prime}$.

It is worth noting that the necessary and sufficient condition (5.6) for the existence of homogeneous deformation fields in the porous material is nothing else than the demand that the stress field in the matrix phase be uniaxial along the direction of the cylindrical pores. This can be expected, since the strain field in the matrix is required to be uniform while no stresses are exerted on the pore surfaces. Furthermore, (5.14) and (5.15) can be viewed as characterizing the admissible strain states of the matrix phase associated to the uniaxial stress state.

Next, applying the formulas (4.5), (4.1) and (4.7), we can exactly evaluate the effective material stress-strain relation at every $\mathbf{E}^{*} \in M$ and the effective 
nominal stress-strain relation and strain-energy function at each $\mathbf{F}^{*} \in M$ for the porous material through

$$
\begin{aligned}
\overline{\mathbf{S}}= & \hat{\mathbf{S}}\left(\mathbf{E}^{*}\right)=c^{(1)} \mathbf{S}^{(1)}\left(\mathbf{E}^{*}\right) \\
= & 2 c^{(1)}\left(\alpha_{2}+\alpha_{3} J_{1}^{*}\right) \mathbf{I}-2 c^{(1)} \alpha_{3} \mathbf{C}^{*}+2 c^{(1)} \\
& \times\left[\alpha_{1}\left(J_{3}^{*}-\sqrt{J_{3}^{*}}\right)-\alpha_{2}-2 \alpha_{3}\right] \mathbf{C}^{*-1}, \\
\overline{\mathbf{P}}= & \hat{\mathbf{P}}\left(\mathbf{F}^{*}\right)=c^{(1)} \mathbf{P}^{(1)}\left(\mathbf{F}^{*}\right) \\
= & 2 c^{(1)}\left(\alpha_{2}+\alpha_{3} J_{1}^{*}\right) \mathbf{R} \mathbf{U}^{*}-2 c^{(1)} \alpha_{3} \mathbf{R} \mathbf{U}^{* 3} \\
& +2 c^{(1)}\left[\alpha_{1}\left(J_{3}^{*}-\sqrt{J_{3}^{*}}\right)-\alpha_{2}-2 \alpha_{3}\right] \mathbf{R} \mathbf{U}^{*-1}, \\
\hat{w}\left(\mathbf{F}^{*}\right)= & c^{(1)} w^{(1)}\left(\mathbf{F}^{*}\right) \\
= & c^{(1)}\left[\alpha_{1}\left(\sqrt{J_{3}^{*}}-1\right)^{2}-\left(\alpha_{2}+2 \alpha_{3}\right) \ln J_{3}^{*}+\alpha_{2}\left(J_{1}^{*}-3\right)+\alpha_{3}\left(J_{2}^{*}-3\right)\right],
\end{aligned}
$$

where use is made of (5.4), (5.5) and (5.1). Substituting the expressions of $\mathbf{C}^{*}$ and $\mathbf{U}^{*}$ specified by (5.14)-(5.15) and the expressions of $J_{i}^{*}$ specified by

$$
J_{1}^{*}(t)=\lambda_{1}^{2}(t)+2 t^{2}, \quad J_{2}^{*}=2 \lambda_{1}^{2}(t) t^{2}+t^{4}, \quad J_{3}^{*}=\lambda_{1}^{2}(t) t^{4},
$$

into (5.17)-(5.19), we obtain

$$
\begin{aligned}
& \overline{\mathbf{S}}=2 c^{(1)}\left(\alpha_{1} t^{4}+2 \alpha_{3} t^{2}+\alpha_{2}-\frac{\alpha_{1} t^{2}}{\lambda_{1}}-\frac{\alpha_{2}+2 \alpha_{3}}{\lambda_{1}^{2}}\right) \mathbf{e}_{1} \otimes \mathbf{e}_{1}, \\
& \left.\overline{\mathbf{P}}=2 c^{(1)}\left[\left(\alpha_{1} t^{4}+2 \alpha_{3} t^{2}+\alpha_{2}\right) \lambda_{1}-\alpha_{1} t^{2}-\frac{\alpha_{2}+2 \alpha_{3}}{\lambda_{1}}\right)\right]\left(\mathbf{R}^{*} \mathbf{e}_{1}\right) \otimes \mathbf{e}_{1}, \\
& \hat{w}\left(\mathbf{F}^{*}\right)=c^{(1)}\left[\alpha_{1}\left(\lambda_{1} t^{2}-1\right)^{2}-\left(\alpha_{2}+2 \alpha_{3}\right) \ln \left(\lambda_{1}^{2} t^{4}\right)\right] \\
& \quad+c^{(1)}\left[\alpha_{2}\left(\lambda_{1}^{2}+2 t^{2}-3\right)+\alpha_{3}\left(2 \lambda_{1}^{2} t^{2}+t^{4}-3\right)\right],
\end{aligned}
$$

where $\lambda_{1}$ is given by $(5.15)_{1}$ and $\mathbf{R}^{*} \in S O(3)$.

As expected, the effective behavior of the porous material evaluated at $\mathbf{E}^{*} \in$ $M$ or $\mathbf{F}^{*} \in M^{\prime}$ is identical to the one of the matrix with the volume fraction $c^{(1)}$ as a multiplier. So, under the surface loading (2.6) with $\overline{\mathbf{F}}=\mathbf{F}^{*} \in M^{\prime}$, the porous 


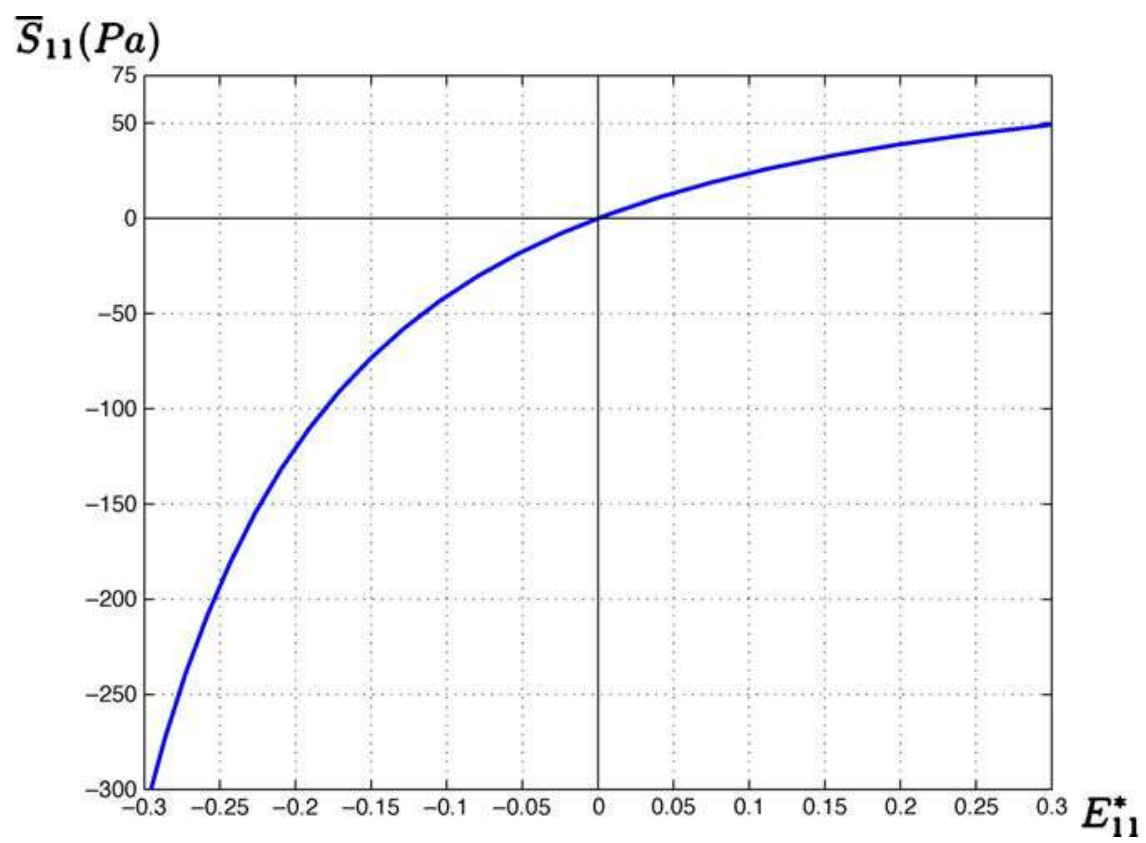

Figure 2. Effective material stress-strain relation $\bar{S}_{11}\left(E_{11}^{*}\right)$ along the direction of cylindrical pores.

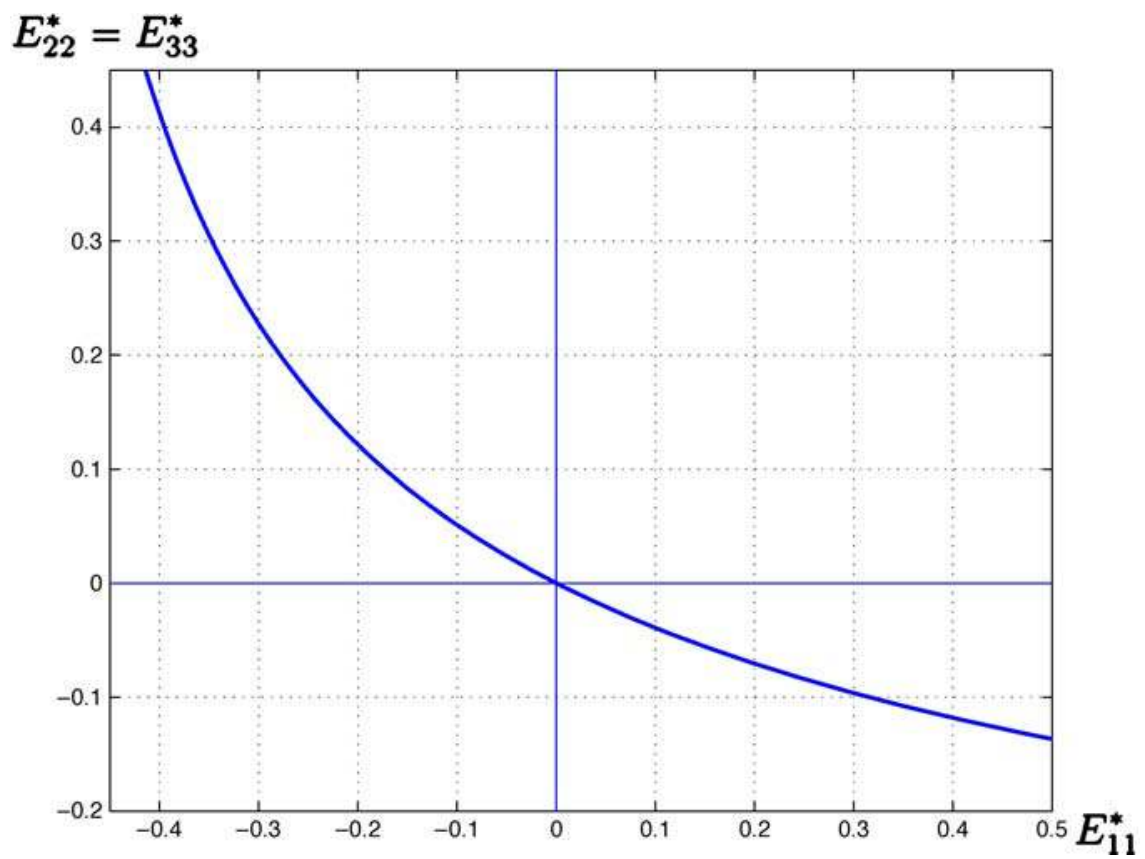

Figure 3. Ratio of the uniform longitudinal strain $E_{11}^{*}$ to the uniform transverse strain $E_{22}^{*}$. 
material under consideration behaves macroscopically as a compressible Mooney-Rivlin material. To graphically represent the relations (5.15) and (5.21), we take the numerical values

$$
c^{(1)}=0.75, \quad \alpha_{1}=500 P a, \quad \alpha_{2}=50 P a, \quad \alpha_{3}=20 P a
$$

for the material parameters of the matrix. The effective material stress-strain relation $\bar{S}_{11}\left(E_{11}^{*}\right)$ along the direction of the cylindrical pores is shown in Figure 2, and the ratio $E_{11}^{*} / E_{22}^{*}$ is illustrated in Figure 3.

After determining all homogeneous deformation fields through (5.14) and (5.15), the exact connections (4.23)-(4.24) or (4.27)-(4.28) can be in principle specified for the porous material in question with no difficulties. However, the corresponding explicit expressions are algebraically cumbersome. Therefore, we do not detail them here.

\section{Final Remarks}

In this work, the important results provided by Hill [14] and Dvorak [7] have been generalized to elastic solids reinforced by elastic fibers, or weakened by unidirectional cylindrical pores, and subjected to large deformations. The method elaborated to accomplish this generalization can be directly used to extend to the case of large deformations other microstructure-independent relations, such as the well-known Levin's one and Rosen-Hashin's one (see, e.g., [22]), established within the framework of linear elasticity or thermoelasticity and on the basis of the concept of uniform fields. Indeed, the main difficulty encountered in doing these extensions in the context of large deformations is due to the dilemma pointed out at the beginning of the paper. The method proposed in this work and consisting in alternatively exploiting the nominal and material description has turned out to be very efficient to solve the dilemma.

All the results derived in Sections 2-5 are relative to a representative element (RVE) $\Omega$ and hence valid for the homogenization of elastic fiber-reinforced composites under consideration. It is interesting to note that all these results remain valid even when $\Omega$ is smaller than an RVE, under the condition that the volume fractions of the fibers and matrix in $\Omega$ correspond to those prescribed for the composite. In this case, the overall properties of $\Omega$ should not be considered as 'effective or homogenized' but 'apparent' (see, e.g., [11, 17]).

As pointed out in the Introduction, an important issue which has not been addressed in this work is that of microscopic and macroscopic instabilities due to the non-convexity of the energy function of an elastic material at finite strain. All the results given in Sections 2-5 are valid only before the occurrence of microscopic instability. In other words, they are meaningful only within the microscopic stability domain. Even though the determination of the latter is an open problem for composites with random microstructures, we conjecture that, 
except in some very special cases, the microscopic stability domain in the space of Green-Lagrange strain tensors $\mathbf{E}$ is in general a domain containing the zero strain tensor $\mathbf{E}=\mathbf{0}$ as an interior point. This conjecture is strongly supported by the recent results of Lopez-Pamies and Ponte Castañeda [19]. Thus, we believe that the results obtained in this work are useful for the homogenization of fiberreinforced composites undergoing large deformations.

\section{Appendix A. Solution of the System of Equations (5.9)-(5.13)}

First of all, note that any physically meaningful solution for the system of nonlinear equations (5.9)-(5.13) must be such that the tensor $\mathbf{C}$ is positive definite, and that every material constant $\alpha_{i}$ involved in (5.9)-(5.13) is such that $\alpha_{i} \geq 0$.

Then, we begin with the simplest equation (5.11). Satisfaction of the latter implies that $C_{12}=0$ or $C_{13}=0$. Without loss of generality, assume that $C_{13}=0$. Then, equation (5.10) is immediately verified and equation (5.9) leads to $C_{12}=0$ or $C_{33}=-\alpha_{2} / \alpha_{3}$. The last expression for $C_{33}$ is not admissible, since it is in contradiction with the requirement that $\mathbf{C}$ be positive definite. So, at this stage, we can conclude that

$$
C_{12}=C_{13}=0 .
$$

This conclusion allows us to simplify (5.12) and (5.13) into

$$
\begin{aligned}
& \alpha_{1}\left(C_{11} C_{22} C_{33}-\sqrt{C_{11} C_{22} C_{33}}\right)+\alpha_{2} C_{22}+\alpha_{3}\left(C_{11}+C_{33}\right) C_{22}-\alpha_{2}-2 \alpha_{3}=0, \\
& \alpha_{1}\left(C_{11} C_{22} C_{33}-\sqrt{C_{11} C_{22} C_{33}}\right)+\alpha_{2} C_{33}+\alpha_{3}\left(C_{11}+C_{22}\right) C_{33}-\alpha_{2}-2 \alpha_{3}=0 .
\end{aligned}
$$

Subtracting (A.3) from (A.2) gives

$$
\left(\alpha_{2}+\alpha_{3} C_{11}\right)\left(C_{22}-C_{33}\right)=0 .
$$

The solution $C_{11}=-\alpha_{2} / \alpha_{3}$ is not admissible and, hence, the following one must hold:

$$
C_{22}=C_{33} \text {. }
$$

Next, introducing (A.4) into (A.2) results in

$$
\left(\alpha_{3}+\alpha_{1} C_{22}\right) C_{22} C_{11}-\alpha_{1} C_{22} \sqrt{C_{11}}+\alpha_{3} C_{22}^{2}+\alpha_{2} C_{22}-\alpha_{2}-2 \alpha_{3}=0 .
$$


This is a quadratic equation of $C_{11}^{1 / 2}$ and has the following admissible solution:

$$
\sqrt{C_{11}}=\frac{\alpha_{1} C_{22}+\sqrt{\alpha_{1}^{2} C_{22}^{2}-4 C_{22}\left(\alpha_{3}+\alpha_{1} C_{22}\right)\left[\alpha_{3}\left(C_{22}^{2}-2\right)+\alpha_{2}\left(C_{22}-1\right)\right]}}{2\left(\alpha_{3}+\alpha_{1} C_{22}\right) C_{22}} .
$$

Combining (5.7), (A.1), (A.4) and (A.6), we finally reach the conclusion that the system of equations (5.9)-(5.13) admits only the solution specified by (5.14) and (5.15) where $C_{11}^{1 / 2}=\lambda_{1}$ and $C_{22}^{1 / 2}=\lambda_{2}=t$ with $t \geq 0$.

\section{References}

1. R. Abeyaratne and N. Triantafyllidis, An investigation of localization in a porous elastic material using homogenization theory. ASME J. Appl. Mech. 51 (1984) 481-486.

2. J.M. Ball, Convexity conditions and existence theorems in nonlinear elasticity. Arch. Rational Mech. Anal. 63 (1977) 337-403.

3. R.B. Borden, A Course in Advanced Calculus. Dover, New York (1998).

4. P. Chadwick and R.W. Ogden, A theorem of tensor calculus and its application to isotropic elasticity. Arch. Rational Mech. Anal. 44 (1971) 54-68.

5. P.G. Ciarlet, Mathematical Elasticity - Volume I: Three-Dimensional Elasticity. NorthHolland, Amsterdam (1988).

6. A. Curnier, Computational Methods in Solid Mechanics. Kluwer, Dordrecht (1994).

7. G.J. Dvorak, On uniform fields in heterogeneous media. Proc. R. Soc. Lond. A 431 (1990) 89-110.

8. G. Geymonat, S. Müller and N. Triantafyllidis, Homogenization of nonlinear elastic materials, microscopic bifurcation and macroscopic loss of rank-one convexity. Arch. Rational Mech. Anal. 122 (1993) 231-290.

9. M.E. Gurtin, An Introduction to Continuum Mechanics. Academic, Boston (1981).

10. Z. Hashin, Large isotropic elastic deformations of composite and porous media. Int. J. Solids Structures 21 (1985) 711-720.

11. Q.C. He, Effects of size and boundary conditions on the yield strength of heterogeneous materials. J. Mech. Phys. Solids 49 (2001) 2557-2575.

12. Q.C. He, Uniform strain fields and microstructure-independent relations in nonlinear elastic fibrous composites. J. Mech. Phys. Solids 47 (1999) 1781-1793.

13. Q.C. He and A. Curnier, A more fundamental approach to damaged elastic stress-strain relations. Int. J. Solids Structures 32 (1995) 1433-1457.

14. R. Hill, Theory of mechanical properties of fiber-strengthened materials - I. Elastic behavior. J. Mech. Phys. Solids 12 (1964) 199-212.

15. R. Hill, On constitutive macro-variables for heterogeneous solids at finite strain. Proc. R. Soc. London A 326 (1972) 131-147.

16. G.A. Holzapfel, Nonlinear Solid Mechanics: A Continuum Approach for Engineering. Wiley, Chichester (2000).

17. C. Huet, Application of variational concepts to size effects in elastic heterogeneous bodies. $J$. Mech. Phys. Solids 38 (1990) 813-841.

18. O. Lopez-Pamies and P.P Castañeda, Second-order estimates for the large-deformation response of particle-reinforced rubbers. C. R. Mecanique 331 (2003) 1-8. 
19. O. Lopez-Pamies and P.P. Castañeda, Second-order estimates for the macroscopic response and loss of ellipticity in porous rubbers at large deformations. J. Elasticity 76 (2004) 247287.

20. C. Miehe, Computational micro-to-macro transitions for discretized micro-structures of heterogeneous materials at finite strains based on the minimization of averaged incremental energy. Comput. Methods. Appl. Mech. Eng. 192 (2003) 559-591.

21. C. Miehe, J. Schröder and M. Becker, Computational homogenization analysis in finite elasticity: Material and structural instabilities on the micro- and macro-scales of periodic composites and their interaction. Comput. Methods. Appl. Mech. Eng. 191 (2002) 4971-5005.

22. G.W. Milton, The Theory of Composites. Cambridge University Press, Cambridge (2002).

23. G.W. Milton and S.K. Serkov, Bounding the current in nonlinear conducting composites. J. Mech. Phys. Solids 48 (2000) 1295-1324.

24. S. Müller, Homogenization of nonconvex integral functionals and cellular elastic materials. Arch. Rational Mech. Anal. 99 (1987) 189-212.

25. S. Nemat-Nasser, Averaging theorems in finite deformation plasticity. Mech. Mater. 31 (1999) 493-523.

26. S. Nemat-Nasser and M. Hori, Micromechanics: Overall Properties of Heterogeneous Materials. Elsevier, Amsterdam (1999).

27. R.W. Ogden, On the overall moduli of non-linear elastic composite materials. J. Mech. Phys. Solids 22 (1974) 541-553.

28. R.W. Ogden, Extremum principles in non-linear elasticity and their application to composites - I. Theory. Int. J. Solids Structures 14 (1978) 265-282.

29. R.W. Ogden, Nonlinear Elastic Deformations. Ellis Horwood and Wiley, Chichester (1984).

30. P. Ponte Castañeda, Second-order homogenization estimates for nonlinear composites incorporating field fluctuations. I. Theory. J. Mech. Phys. Solids 50 (2002) 737-757.

31. P.P. Castañeda and P. Suquet, Nonlinear composites. In: E. van der Giessen and T.W. Wu (eds.). Advances in Applied Mechanics, vol. 34, Academic, San Diego (1998) pp. 171-302.

32. S. Torquato, Random Heterogeneous Media: Microstructure and Macroscopic Properties. Springer, Berlin Heidelberg New York (2001).

33. N. Triantafyllidis and B.N. Maker, On the comparison between microscopic and macroscopic instability mechanisms in a class of fiber-reinforced composites. ASME J. Appl. Mech. 52 (1985) 794-800.

34. C. Truesdell and W. Noll, The Non-Linear Field Theories of Mechanics. Springer, Berlin Heidelberg New York (1992). 